\title{
Nonlinear Interaction of Seismic Waves in the Lab: A potential Tool for Characterizing Pore Struc- ture and Fluids
}

\author{
Thomas Gallot, Alison Malcolm*, Earth Resources Laboratory, MIT, T. L. Szabo, Biomedical Engineering, Boston \\ University, Dan Burns, Stephen Brown, Michael Fehler, Earth Resources Laboratory, MIT
}

\section{SUMMARY}

As more and more resources are extracted from unconventional reservoirs, an understanding of the microstructure of reservoir rocks is of increasing importance. Many conventional techniques struggle to sense variations in the micro-structure and pore-fluids of rock samples. The nonlinear coupling of two elastic waves is known to be sensitive to these parameters, however, and so is a natural candidate to improve our understanding of these structures. Here, we develop an experimental technique to sense the nonlinear interaction of two propagating waves: a strong $\mathrm{S}$-wave pump that changes (minutely) the elastic properties of the sample and a weaker P-wave probe that senses those changes. By measuring the delay in the $\mathrm{P}$-wave probe traveltime induced by the S-wave pump, we show that this signal is significant in a Berea sandstone sample and absent in Aluminum and Plexiglass samples. The polarization of the S-wave (particle motion aligned or perpendicular to the P-wave probe) has a large impact on the measured response; this is evidence that the signal we measure is sensitive to the microstructure of the rock. We show that the method is sensitive to fluids by imaging the variations in two specific nonlinear parameters, caused by the introduction of fluid into a Berea sandstone sample.

\section{INTRODUCTION}

The production of unconventional resources relies on permeability in fractures. The characterization of such fractures, and the fluids within them, is thus becoming more and more important. In addition to their impact on anisotropy (Thomsen, 1995), both fractures and fluids contribute to the nonlinear elastic response of a rock (Guyer and Johnson, 1999; Guyer et al., 1999; Guyer and Johnson, 2009). Because of this, imaging the nonlinear parameters may improve our understanding of this sort of resource.

The laboratory experiment described here is a first step toward this goal. Building on work done in the resonance regime in medical imaging, (Muller et al., 2005; Renaud et al., 2008, 2009) and for rock samples in (Johnson et al., 1991; D Angelo et al., 2004; Renaud et al., 2012), we develop and test an experimental setup designed to image changes in the nonlinear parameters throughout a sample. In our experiment, we measure the non-linearity through a change, caused by a propagating $\mathrm{S}$-wave, in the traveltime of a propagating $\mathrm{P}$-wave. In other words, we look at the nonlinear interaction between two waves: a low-amplitude P-wave probe that senses the change caused by a highamplitude S-wave pump. (We consider the pump to be changing the elastic properties of the rock and the probe as sensing that change; this probe/pump terminology is discussed further in Rivière et al. (2013).)

That nonlinear responses can be recorded in the field is established, although most work has been done on soils (Johnson et al., 2009; Lawrence et al., 2009; Cox et al., 2009), or at crustal scales (Rivet et al., 2011). The closest work to that done here is the Dynamic Acousto-Elastic Testing method developed in (Renaud et al., 2012), which has been tested in the field in (Renaud et al., 2013).

We first establish that we are able to measure the change in P-wave traveltime caused by the $\mathrm{S}$-wave, in a Berea sandstone sample in the lab. We look only at 'direct' waves, i.e. waves that have not reflected from the boundaries of the sample, and assume that the sample is homogeneous and isotropic. (Measurements put the P-wave anisotropy at approximately $1 \%$.) A related experimental procedure is discussed

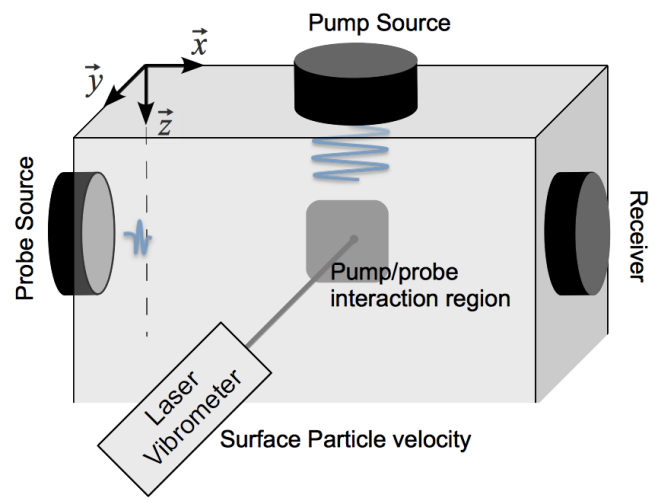

Figure 1: Experimental setup. The S-wave pump (top transducer) and P-wave probe (left transducer) propagate into the material and interact at approximately the location of the laser beam. The signals are recorded on the receiver (right transducer) and the delay in the probe traveltime is measured (see Figure 2) as a function of the phase delay $\phi$ between the pump and probe. The data recorded with the laser are used to calibrate the experiment and estimate the strain.

\begin{tabular}{|c|c|}
\hline Sample & \\
\hline$V_{p}$ & $2450 \mathrm{~m} / \mathrm{s}$ \\
$V_{s}$ & $1550 \mathrm{~m} / \mathrm{s}$ \\
$\rho$ & $2700 \mathrm{~kg} / \mathrm{m}^{3}$ \\
Elastic modulus & $M=16 \mathrm{GPa}$ \\
Size & $15 \times 15 \times 3 \mathrm{~cm}$ \\
\hline Experimental & \\
\hline$f_{\text {pump }}$ & $50 \mathrm{kHz}$ \\
$f_{\text {probe }}$ & $500 \mathrm{kHz}$ \\
\hline
\end{tabular}

Table 1: Experimental and sample parameters

from a theoretical perspective in (Belyayeva et al., 1994), but to our knowledge it has not yet been performed in the laboratory. We then apply this experimental procedure to two tests. First, we explore the relationship between the polarization of the S-wave pump and the magnitude of the nonlinear response. Second, we use a setup analogous to a crosswell tomography experiment in our laboratory sample, and attempt to localize a change in the nonlinear properties induced by the introduction of fluid into the sample.

\section{EXPERIMENTAL DESIGN}

The goal of our experiment is to measure the traveltime delay in a low-amplitude wave (the probe) caused by a high-amplitude wave (the pump). This sort of wave-wave interaction is inherently nonlinear and so measuring the magnitude of the traveltime delay gives us an idea of the strength of the non-linearity. The experimental setup is shown in Figure 1. We use a sample of Berea sandstone; the sample is nearly isotropic, with moderate porosity and permeability. (The experimental 


\section{Nonlinear pore structure characterization}

and sample parameters are given in Table 1.) The pump wave is excited with a Panametrics transducer that is glued to the top sample for most experiments. The probe transmitter is glued to the side of the sample. All signals are recorded on the same receiving transducer glued to the opposite side of the sample from the probe source.

This experiment looks at the interaction of two traveling waves, in contrast to work such as Dynamic Acousto-Elastic Testing (Renaud et al., 2012), in which changes are sensed in a sample at resonance. For the traveling waves to interact, they must travel through the same part of the rock at the same time. We achieve this by triggering the two signals with a known time delay, $\phi$, between them; this time delay is then computed to be such that the two signals overlap. (Of course there is interaction along the entire probe path, but we expect this to be strongest in the region marked on Figure 1.)

In order to quantify the change in the probe signal induced by the pump, the pump signal should be slowly varying compared to that of the probe. In other words, the pump should not change phase (too much) while the probe is interacting with it. To ensure that this is the case, we use a $500 \mathrm{kHz}$ probe wave and a $50 \mathrm{kHz}$ pump. This difference in frequency allows us to assume that the pump wave does not change significantly during its interaction with the probe at each delay $\phi$. We then vary the delay, $\phi$, between the two signals through several periods of the pump wave to observe the induced changes on the probe caused by both directions (i.e. $\pm x$ ) of particle motion. We use an Swave pump for two reasons. First, to have the particle motions of the (P-wave) probe and pump aligned and second to be able to (relatively) easily vary the particle motion by rotating the pump transducer.

The nonlinear effect we are looking for is small. The delays shown below are on the order of a few tens of nanoseconds; the time for the P-wave probe to travel across the sample is about $65 \mu \mathrm{s}$, so we are measuring a delay that is $0.1 \%$ of the total traveltime. Although making measurements this small in the field would be challenging, it is not outside the realm of possibility. In order to extract such a small signal, we developed the following procedure, illustrated in Figure 2:

1. record the pump signal (black), $S_{1}$

2. record the probe signal with the pump off (blue), $S_{2}$

3. record the wave mixing signal (red) (pump and probe both on), $S_{3}$

4. subtract $S_{1}$ from $S_{3}$ to obtain an estimate of the perturbed pump signal (green), $S_{4}$

5. cross-correlate the original probe signal, $S_{2}$ with the perturbed probe signal, $S_{4}$ to obtain an estimated traveltime delay.

From this sequence of steps we obtain a single measurement of the time-modulation of the probe pulse. We denote this measurement by $T M(\phi)$ and repeat the experiment for $\phi$ ranging through several periods of the pump signal. As discussed below, we estimate the strain induced by the pump to be $\approx 10^{-5}-10^{-6}$ and that of the probe to be $\approx 10^{-8}$. (Note that the driving amplitude of the pump is significantly amplified to achieve the larger strain.) These numbers lead us to believe that we can expect the pump to be inducing nonlinear effects and the probe not to be. This is exactly what we would like; the $\mathrm{S}$-wave pump is changing the elastic properties of the sample, while the $\mathrm{P}$-wave probe is not.

\section{EXPERIMENTAL RESULTS}

To verify that the response we record comes from the rock itself and is not an artifact of the experimental setup, we perform the experiment for three different materials, as shown in Figure 3. Both Aluminum and Plexiglas are expected to be linear materials and we indeed see
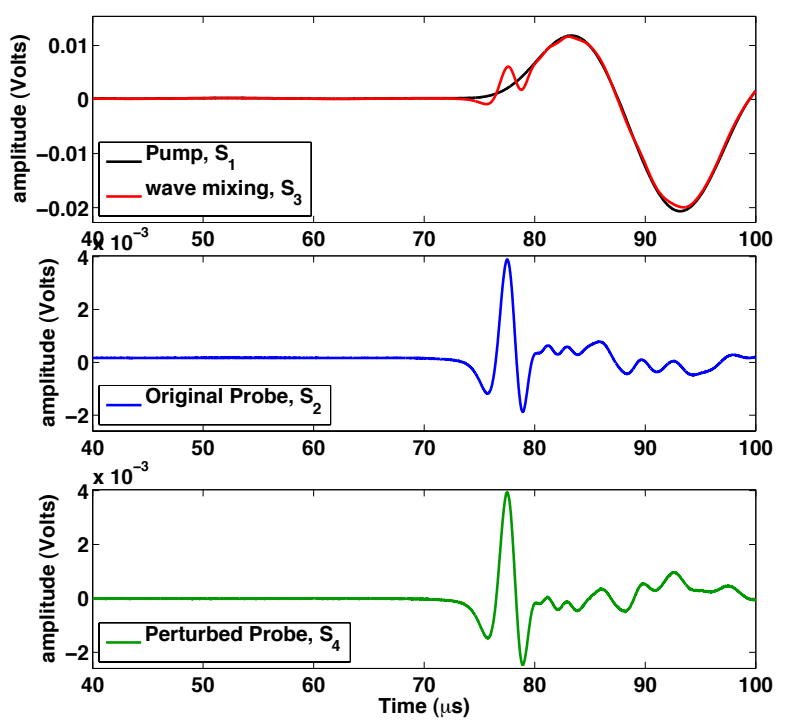

Figure 2: To measure the delay times, we record three signals: the probe alone (original probe: blue, $S_{2}$ ) the pump alone (black, $S_{1}$ ), the pump and probe together (wave mixing: red, $S_{3}$ ). The second signal is then subtracted from the first $\left(S_{3}-S_{1}\right)$ to obtain the perturbed probe signal (green, $S_{4}$ ). This signal (green, $S_{4}$ ) is then cross-correlated with the original probe (blue, $S_{1}$ ) and a delay time is obtained from the shift in the peak of the crosscorrelation.

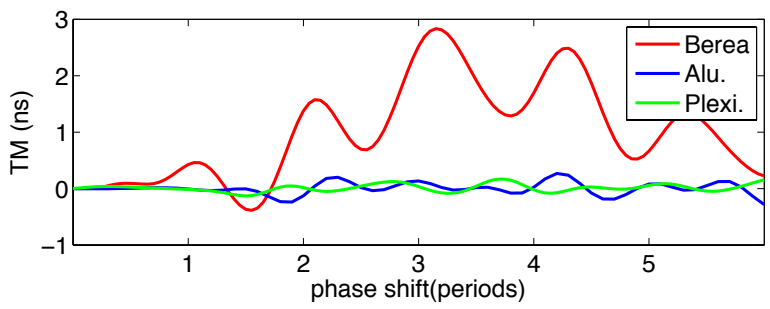

Figure 3: Data for initial experiment; the time modulation, measured with the correlation procedure described in the text and in Figure 2, as a function of the phase delay between the two pulses. From this figure, we conclude that the nonlinear signal we see is a property of the rock and not of the measurement system; both Plexiglas and Aluminum are expected to be linear materials.

that the measured time modulation in these two materials is significantly smaller than that in the Berea sandstone sample. Note that for this experiment, the data are displayed as a function of phase shift in periods, rather than in $\mu \mathrm{s}$; this is because the different materials have different speeds and thus wavelengths. Using periods allows a more direct comparison.

Figure 4 shows the experimental results for the Berea sandstone described above, for six cycles of the pump, with several small modifications to increase the pump amplitude (and thus that of the delay times) compared to that in Figure 3.

In gray in the top plot, we compare the measured pump signal to a linear elastic model. On the same plot in black, we plot the measured traveltime delay $T M$ as a function of $\phi$, (the phase difference between the pump and probe waves). The delay time measurements contain 


\section{Nonlinear pore structure characterization}
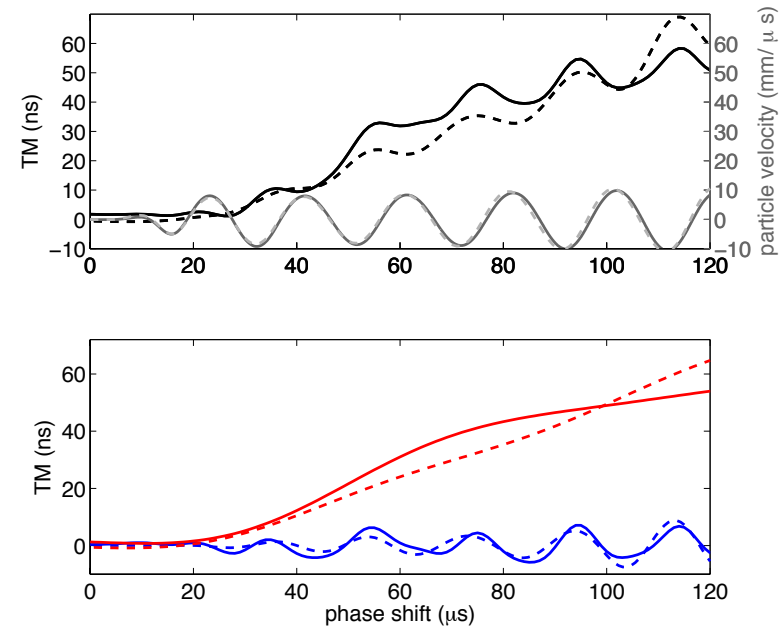

Figure 4: Top: Recorded data in Berea sandstone sample (solid black line) along with fit using equation 4 (dashed black line), to recover $\beta=-542$ and $\delta=-3 \times 10^{9}$. In gray are the measured (solid) and modeled (dashed) particle velocity measured with the laser vibrometer on the surface of the sample in the wave-interaction region. Bottom: Data from top plot filtered to highlight the two scales of variation. The fast varying (blue) curve is fit to obtain $\beta$ and the slowly varying (red) curve is fit to obtain $\delta$

signal at different frequencies; we show the filtered signal in each of these frequency bands in the lower plot. The higher frequency signal is at the same frequency as the pump wave itself (compare the blue signal in the lower plot with the measured pump signal in gray on the top plot), while the second is much lower frequency. This suggests that we will require at least two parameters to fit the data. This is discussed further below.

One of the most important observations of the signals in Figure 4 is that there are no values of the phase delay $\phi$ that result in an increase in velocity of the P-wave probe, i.e. all recorded time shifts are delays in the probe arrival time. This indicates that the $\mathrm{S}$-wave pump is acting to soften the rock. We see the effect of the frequency of the pump in the higher-frequency signal, but the magnitude of the low-frequency signal ensures that we record only delays. We initially believed that this effect could come from so-called slow-dynamics (Ten Cate and Shankland, 1996), but a careful study of the effect of the time between measurements proved that this is not the case (Gallot et al., 2014). Our current hypothesis is that this softening is caused by a hysteresis in the response of cracks to the passing S-wave (e.g. Holcomb, 1981; Scholz and Hickman, 1983)

\section{Recovering Nonlinear Parameters, $\beta \& \delta$}

As noted above, the dual-frequency nature of the measured data suggest that two parameters will be required to explain them. In this section, we describe two such parameters, $\beta$ and $\delta$ described in more detail below. These parameters are well-established in the nonlinear characterization of materials, and are reported in (Renaud et al., 2012) for a variety of samples and strain regimes. They were also measured in static experiments in the laboratory in (Hughes and Kelly, 1953). To explain the nature of these parameters, we give a very brief derivation, more details can be found in (McCall, 1994; Van Den Abeele, 1996) For static strains in one dimension, these parameters can be related to the third-order elastic constants (Landau and Lifshitz, 1986, p 107).

The root of the nonlinear wave interactions comes from a nonlinear relationship between stress and strain. More specifically, rather than a traditional Hooke's law

$$
\sigma=M \varepsilon
$$

in which $\sigma$ is the stress, $M$ is an elastic modulus, and $\varepsilon$ is the strain, we use

$$
\sigma=M\left(\varepsilon+\beta \varepsilon^{2}+\delta \varepsilon^{3}\right),
$$

where $\beta$ and $\delta$ are the nonlinear parameters we hope to recover.

In order to recover $\beta$ and $\delta$, we need an estimate of $\varepsilon$. We cannot measure the strain directly in-situ and so we resort to the following method to obtain an estimate:

1. Measure the particle velocity, $v_{\text {meas }}$ on the surface of the sample with the laser vibrometer as marked in Figure 1

2. Use the signal recorded by the vibrometer in step 1 . as a source in a finite difference model (using the method developed by Virieux (1986); Graves (1996)) to obtain modeled velocity $v_{m o d}$ and stress $\sigma_{m o d}$

3. Scale the modeled velocity $v_{\text {mod }}$, from the finite-difference model in step 2. to match the measured amplitude, $v_{\text {meas }}$ in step 1., obtaining the scaling factor $s$

4. Scale the modeled stress by $s$

5. Use a linear Hooke's law to compute the strain $\varepsilon_{m o d}$, throughout the sample.

Although we do not expect this method to be extremely accurate, we expect the distribution and order of magnitude of the strain to be well estimated. It is from this procedure that we estimate that $\varepsilon \approx 10^{-8}$ for the probe and $10^{-5}$ or $10^{-6}$ for the pump.

With the estimated strain, we can now obtain an estimate of $\beta$ and $\delta$. To do this, we must first relate the time modulation, $T M$ to $\sigma$. As detailed in (Gallot et al., 2014; Renaud et al., 2011) this can be done using the two expressions

$$
\begin{aligned}
d T & =\frac{-d l}{2 c} \frac{d M}{M} \\
\frac{d M}{M} & =\beta \varepsilon+\delta \varepsilon^{2},
\end{aligned}
$$

where $T$ is the traveltime along the path, $l$ is the path length and $M$ is the elastic modulus. There is an ambiguity in the above equation coming from the fact that the strain is represented here as a scalar. This is a relic of the 1D nature of the original theory; from a theoretical viewpoint it is not completely clear what should be used. We choose the total strain (trace of the strain tensor), integrated along the probe path and averaged over the pump beam width. We choose the total strain because it stands to reason that a compressional wave will sense strain in all directions. We integrate along the probe path because we have a non-planar probe excitation and thus the strain distribution changes along the path. We average over the beam width of the pump to compensate for the finite frequency of both the pump and probe.

The nonlinear parameters, $\beta$ and $\delta$ are then obtained by fitting the recorded time modulation to the calculated strains. Note that because the input signal is $\propto \cos \omega_{\text {pump }} t$ the strain is also $\propto \cos \omega_{\text {pump }} t$ and $\varepsilon^{2} \propto 1+\cos 2 \omega_{\text {pump }} t$ allowing us to separately estimate $\beta$ and $\delta$ by appropriate filtering. The fits obtained in this way are shown in Figure 4 . We see that despite the many approximations made the theory does account for most of the measured signal. We note also that our recovered values of $\beta=-542$ and $\delta=-3 \times 10^{9}$ are in general agreement with those reported in (Renaud et al., 2012) for similar samples. Understanding the implications of these assumptions, as well as the interpretation of what the values of $\beta$ and $\delta$ mean (keeping in mind they are strain dependent) is a subject of current work.

\section{Changing the Polarization of the Pump}

Although the exact mechanisms causing non-linearity are not clearly understood, it is clearly observed that damaged, fluid-filled, samples 


\section{Nonlinear pore structure characterization}
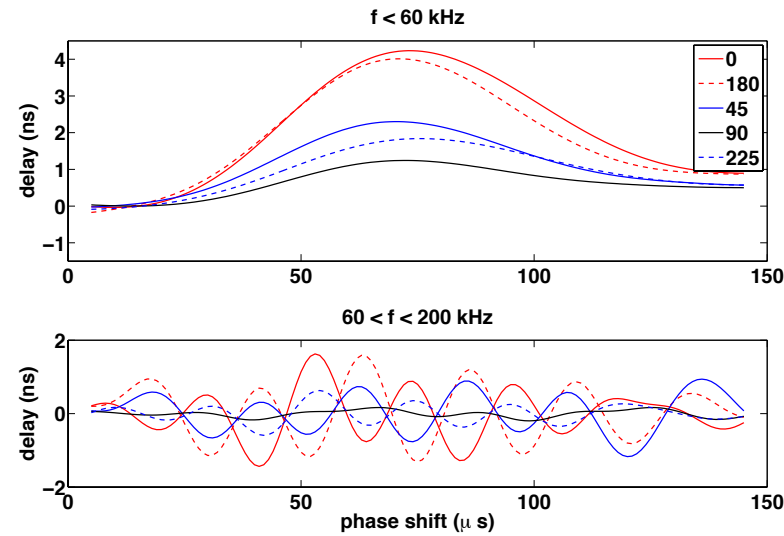

Figure 5: Measured delay times for different pump polarizations. The legend gives the angle (in degrees) between the particle motion of the pump and probe waves; the probe wave particle motion is always along the $x$-axis. The magnitude of the measured delay times decreases when the polarizations are not aligned; the differences may be caused by differences in the pump wave's effect on the microstructure of the rock.

are more strongly nonlinear (Guyer and Johnson, 2009). Because of this, we expect the magnitude of the nonlinear signal to be correlated with micro-crack structure as well as fluid content. We therefore expect that changing the relative orientation of the particle motions of the pump and probe will change the strength of the nonlinear interaction. To explore this, we repeat the experiment described above with four cycles of different polarizations of the S-wave pump.

In the experiments described in the previous sections, we glue the transducers to the sample in order to ensure, and maintain, good coupling between the rock and transducer. Looking at different polarizations requires us to physically turn the transducer, however, so instead of gluing it to the sample, we use a shear wave couplant and place a weight on top of the transducer to ensure repeatable coupling.

The results of this experiment are shown in Figure 5. The legend gives the angle between the P-wave particle motion (always along $x$ in Figure 1) and the $\mathrm{S}$-wave particle motion (ranging from along $x\left(0^{\circ}\right.$ and $180^{\circ}$ to along $y\left(90^{\circ}\right)$ ). The data are shown filtered into the same two frequency bands described above for the estimation of $\beta$ and $\delta$. The consistency of the results between $0^{\circ}$ and $180^{\circ}$ shows that we have indeed achieved very consistent coupling of the pump transducer. The decrease in signal magnitude in both frequency bands when the polarization is turned so that the particle motions of the two waves are no longer aligned is a strong indication that the signal is sensing the microstructure. We hypothesize that what is happening is that when the particle motions of the two waves are aligned the high-amplitude $S$ wave pump is perturbing cracks with their normals in the $x$-direction. These are the same cracks that we would expect the P-wave pump to be most sensitive to, thus having the largest effect on its traveltime. The exact perturbation the shear wave induces on these cracks could be an open/closing perturbation, but because it is generating a shear stress and not a tensile stress, it could also be rotating or perturbing the cracks in another way.

\section{A PRELIMINARY IMAGE}

As stated in the introduction, this work is meant to be a stepping stone on the path to forming and interpreting an image of subsurface nonlinearity. To that end, we now test whether our method can be extended to sense regions with different nonlinear properties, $\beta$ and $\delta$. To create a sample in which $\beta$ and $\delta$ are not homogeneous, we introduce a penetrating oil (WD-40) into the original Berea sample at two locations, with different amounts of oil at each location. We then setup a cross-well experiment in which a single source is moved to six differ-

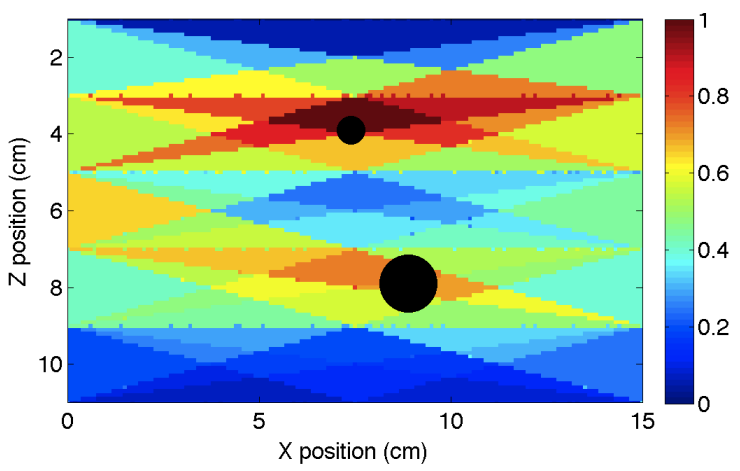

Figure 6: A preliminary (normalized) image made of the delay times using a crosswell-like array of 6 sources and receivers. The measured delays are back-projected along beams to obtain the image seen here; the smearing is a result of the relatively low sampling. The black circles mark the locations at which a small amount of fluid (WD-40) was introduced into the samples.

ent positions on one side of the sample and the response is recorded simultaneously on six transducers glued to the other side of the sample. All other parameters of the experiment remain as described above and summarized in Table 1.

Once we have collected the data set and computed the delays times on each of the 36 source/receiver pairs, we do a simple tomography to estimate the nonlinear parameters throughout the sample. Specifically, we take the measured delays and spread them over the (straight) ray-path from the source receiver as in a very simple iteration of traveltime tomography. The results are shown in Figure 6. Although the result is blurry, we certainly seem to have recovered the locations of the anomalies. This is still a preliminary result, there remain many questions about the origin of the difference and its magnitude. The most pressing of these is the role of attenuation on the result. It is well known that the presence of fluid causes attenuation (e.g. Winkler and Murphy, 1995), and we definitely see a significant change in the recorded signals that may be because of this effect. We are working to understand how much the attenuation effects the quality of our measurements of the nonlinear parameters as well as whether the image made with this nonlinear imaging method gives higher resolution or more information than an image of the attenuation itself. We expect the nonlinear image to be, at the least, more robust, since it is based on traveltime, rather than amplitude, measurements.

\section{CONCLUSIONS}

We have designed an experiment to image the nonlinear parameters, $\beta$ and $\delta$ of an unbounded rock sample. We have shown that it is possible to record the traveltime delay in a P-wave probe caused by a propagating S-wave pump signal in an experiment designed for finite-sized samples. We have given preliminary evidence that the signals are sensitive to the microstructure of the rock, specifically the orientation of cracks. We have further shown that it is possible to do a simple tomography to form a preliminary image of fluid content through the variation of $\beta$ and $\delta$ in the sample. This opens up the possibility of imaging the nonlinear parameters of rocks both in the lab, and in the future in the field.

\section{ACKNOWLEDGMENTS}

We are grateful for the financial support we have received from Weatherford and the Earth Resources Laboratory. We would also like to thank Xinding Fang for his help with the numerical experiments used to estimate the strain in the physical experiments and Ingo Geldmacher and Jim Tencate for helpful discussions. 
http://dx.doi.org/10.1190/segam2014-0248.1

\section{EDITED REFERENCES}

Note: This reference list is a copy-edited version of the reference list submitted by the author. Reference lists for the 2014 SEG Technical Program Expanded Abstracts have been copy edited so that references provided with the online metadata for each paper will achieve a high degree of linking to cited sources that appear on the Web.

\section{REFERENCES}

Belyayeva, I. Y., V. Y. Zaitsev, and A. M. Sutin, 1994, Tomography of elastic nonlinear parameters of rocks in problems of seismology and seismic prospecting: Physics of the Solid Earth: English Translation, 30, no. 12, 1064-1071.

Cox, B. R., I. I. Stokoe, H. Kenneth, and E. M. Rathje, 2009, An in situ test method for evaluating the coupled pore pressure generation and nonlinear shear modulus behavior of liquefiable soils: Geotechnical Testing Journal, 32, no. 1, doi: 10.1520/GTJ101484.

D’Angelo, R. M., K. W. Winkler, T. J. Plona, B. J. Landsberger, and D. L. Johnson, 2004, Test of hyperelasticty in highly nonlinear solids: Sedimentary rocks: Physical Review Letters, 93, no. 21, 214301, http://dx.doi.org/10.1103/PhysRevLett.93.214301.

Graves, R. W., 1996, Simulating seismic wave propagation in 3D elastic media using staggered-grid finite differences: Bulletin of the Seismological Society of America, 86, 1091-1106.

Guyer, R. A., and P. A. Johnson, 1999, Nonlinear mesoscopic elasticity: Evidence for a new class of materials: Physics Today, 52, no. 4, 30-36, http://dx.doi.org/10.1063/1.882648.

Guyer, R. A., and P. A. Johnson, 2009, Nonlinear mesoscopic elasticity: The complex behaviour of granular media including rocks and soil: Wiley-VCH Verlag.

Guyer, R. A., J. Ten Cate, and P. Johnson, 1999, Hysteresis and the dynamic elasticity of consolidated granular materials: Physical Review Letters, 82, no. 16, 3280-3283, http://dx.doi.org/10.1103/PhysRevLett.82.3280.

Holcomb, D. J., 1981, Memory, relaxation, and microfracturing in dilatant rock: Journal of Geophysical Research, 86, no. B7, 6235-6248, http://dx.doi.org/10.1029/JB086iB07p06235.

Hughes, D. S., and J. L. Kelly, 1953, Second-order elastic deformation of solids: Physical Review Letters, 92, 1145-1149.

Johnson, P. A., P. Bodin, J. Gomberg, F. Pearce, Z. Lawrence, and F. Y. Menq, 2009, Inducing in situ, nonlinear soil response applying an active source: Journal of Geophysical Research: Solid Earth, 114, no. B5, doi: 10.1029/2008JB005832.

Johnson, P. A., A. Migliori, and T. J. Shankland, 1991, Continuous wave phase detection for probing nonlinear elastic wave interactions in rocks: The Journal of the Acoustical Society of America, 89, no. 2, 598-603, http://dx.doi.org/10.1121/1.400384.

Landau, L. D., and E. M. Lifshitz, 1986, Theory of elasticity: Elsevier.

Lawrence, Z., P. Bodin, and C. A. Langston, 2009, In situ measurements of nonlinear and nonequilibrium dynamics in shallow, unconsolidated sediments: Bulletin of the Seismological Society of America, 99, no. 3, 1650-1670, http://dx.doi.org/10.1785/0120080177.

McCall, K., 1994, Theoretical study of nonlinear elastic wave propagation: Journal of Geophysical Research: Solid Earth, 99, no. B2, 2951-2600. 
Muller, M., A. Sutin, R. Guyer, M. Talmant, P. Laugier, and P. A. Johnson, 2005, Nonlinear resonant ultrasound spectroscopy (NRUS) applied to damage assessment in bone: The Journal of the Acoustical Society of America, 118, no. 6, 3946-3952, http://dx.doi.org/10.1121/1.2126917.

Renaud, G., S. Callé, and M. Defontaine, 2009, Remote dynamic acoustoelastic testing: Elastic and dissipative acoustic nonlinearities measured under hydrostatic tension and compression: Applied Physics Letters, 94, no. 1, 011905, http://dx.doi.org/10.1063/1.3064137.

Renaud, G., S. Callé, J. P. Remenieras, and M. Defontaine, 2008, Exploration of trabecular bone nonlinear elasticity using time-of-flight modulation: IEEE Transactions on Ultrasonics, Ferroelectrics, and Frequency Control, 55, no. 7, 1497-1507, http://dx.doi.org/10.1109/TUFFC.2008.825.

Renaud, G., P.-Y. Le Bas, and P. A. Johnson, 2012, Revealing highly complex elastic nonlinear (anelastic) behavior of earth materials applying a new probe: Dynamic acoustoelastic testing (DAET): Journal of Geophysical Research: Solid Earth, doi:10.1029/2011JB009127.

Renaud, G., M. Talmant, S. Callé, M. Defontaine, and P. Laugier, 2011, Nonlinear elastodynamics in micro-inhomogeneous solids observed by head-wave based dynamic acoustoelastic testing: The Journal of the Acoustical Society of America, 130, no. 6, 3583-3589, http://dx.doi.org/10.1121/1.3652871.

Rivet, D., M. Campillo, N. M. Shapiro, V. Cruz Atienza, M. Radiguet, N. Cotte, and V. Kostoglodov, 2011, Seismic evidence of nonlinear crustal deformation during a large slow slip event in Mexico: Geophysical Research Letters, 38, L08308.

Rivière, J., G. Renaud, R. A. Guyer, and P. A. Johnson, 2013, Pump and probe waves in dynamic acousto-elasticity: Comprehensive description and comparison with nonlinear elastic theories: Journal of Applied Physics, 114, 054905.

Scholz, C. H., and S. H. Hickman, 1983, Hysteresis in the closure of a nominally flat crack: Journal of Geophysical Research, 88, no. B8, 6501-6504, http://dx.doi.org/10.1029/JB088iB08p06501.

Ten Cate, J. A., and T. J. Shankland, 1996, Slow dynamics in the nonlinear elastic response of Berea sandstone: Geophysical Research Letters, 23, no. 21, 3019-3022, http://dx.doi.org/10.1029/96GL02884.

Thomsen, L., 1995, Elastic anisotropy due to aligned cracks in porous rock: Geophysical Prospecting, 43, no. 6, 805-829, http://dx.doi.org/10.1111/j.1365-2478.1995.tb00282.x.

Van Den Abeele, K. E.-A., 1996, Elastic pulsed wave propagation in media with second or higher-order nonlinearity. Part I. Theoretical framework: The Journal of the Acoustical Society of America, 99, no. 6, 3334-3345, http://dx.doi.org/10.1121/1.414890.

Virieux, J., 1986, $P-S V$ wave propagation in heterogeneous media: Velocity-stress finite-difference method: Geophysics, 51, 889-901, http://dx.doi.org/10.1190/1.1442147.

Winkler, K. W., and W. F. Murphy III, 1995, Acoustic velocity and attenuation in porous rocks, in Rock physics and phase relations: A handbook of physical constants: AGU Reference Shelf, 3, 20-34, http://dx.doi.org/10.1029/RF003p0020. 